ARTICLE

\title{
Quantum-mechanical exploration of the phase diagram of water
}

\author{
Aleks Reinhardt (1) ${ }^{1 凶} \&$ Bingqing Cheng $\mathbb{1}^{2,3 凶}$
}

The set of known stable phases of water may not be complete, and some of the phase boundaries between them are fuzzy. Starting from liquid water and a comprehensive set of 50 ice structures, we compute the phase diagram at three hybrid density-functional-theory levels of approximation, accounting for thermal and nuclear fluctuations as well as proton disorder. Such calculations are only made tractable because we combine machine-learning methods and advanced free-energy techniques. The computed phase diagram is in qualitative agreement with experiment, particularly at pressures $\lesssim 8000$ bar, and the discrepancy in chemical potential is comparable with the subtle uncertainties introduced by proton disorder and the spread between the three hybrid functionals. None of the hypothetical ice phases considered is thermodynamically stable in our calculations, suggesting the completeness of the experimental water phase diagram in the region considered. Our work demonstrates the feasibility of predicting the phase diagram of a polymorphic system from first principles and provides a thermodynamic way of testing the limits of quantum-mechanical calculations.

\footnotetext{
${ }^{1}$ Department of Chemistry, University of Cambridge, Lensfield Road, Cambridge CB2 1EW, UK. ${ }^{2}$ Accelerate Programme for Scientific Discovery, Department of Computer Science and Technology, 15 J.J. Thomson Avenue, Cambridge CB3 OFD, UK. ${ }^{3}$ Cavendish Laboratory, University of Cambridge, J.J. Thomson Avenue, Cambridge CB3 OHE, UK. ®email: ar732@cam.ac.uk; bc509@cam.ac.uk
} 
W ater is the only common substance that appears in all three states of aggregation-gas, liquid and solidunder everyday conditions ${ }^{1}$, and its polymorphism is particularly complex. In addition to hexagonal ice (ice $\mathrm{I}_{\mathrm{h}}$ ) that forms snowflakes, there are currently 17 experimentally confirmed ice polymorphs and several further phases have been predicted theoretically ${ }^{2}$. The phase diagram of water has been extensively studied both experimentally and theoretically over the last century; nevertheless, it is not certain if all the thermodynamically stable phases have been found, and the coexistence curves between some of the phases are not well characterised ${ }^{2}$. At high pressures, experiments become progressively more difficult, and therefore computer simulations play an increasingly crucial role.

Computing the thermodynamic stabilities of the different phases of water is challenging because quantum thermal fluctuations and, in proton-disordered ice phases, the configurational entropy need to be taken into account in free-energy calculations. Considerable insight has been gained into the phase behaviour of water using empirical potentials $s^{3-13}$, which inevitably entail severe approximations ${ }^{3}$. For example, the rigid water models such as TIP $n \mathrm{P}^{14-16}$ and SPC/ $\mathrm{E}^{17}$ cannot describe the fluctuations of the bond lengths and angles and therefore do not explicitly include nuclear quantum effects (NQEs) $)^{18,19}$, although some have been extended to incorporate fluctuations ${ }^{20-22}$. The MB-pol force field ${ }^{23}$, which includes many-body terms fitted to the coupledcluster level of theory, has not been fitted to the high-pressure part of the water phase diagram. Describing the phase diagram is a particularly stringent test for water models, and indeed, only the TIP4P-type models ${ }^{14-16}$ and the iAMOEBA water model ${ }^{24}$ reproduce the qualitative picture, while the SPC/E, TIP3P and TIP5P models predict ice $I_{h}$ to be stable only at negative pressures 25,26

A promising route for predicting phase diagrams is from electronic structure methods (i.e. ab initio), but combining these methods with free-energy calculations is extremely expensive. However, machine-learning potentials (MLPs) have emerged as a way of sidestepping the quantum-mechanical calculations by using only a small number of reference evaluations to generate a data-driven model of atomic interactions ${ }^{27}$. As an example, MLPs have been employed to reveal the influence of Van der Waals corrections on the thermodynamic properties of liquid water ${ }^{28}$. Later, a similar framework, also employing accurate reference data at the level of hybrid density-functional theory (DFT), reproduced several thermodynamic properties of solid and liquid water at ambient pressure ${ }^{29}$. Very recently, multithermal-multibaric simulations were used to compute the phase diagram and nucleation behaviour of gallium ${ }^{30}$, while simulations using MLPs provided evidence for the supercritical behaviour of high-pressure hydrogen ${ }^{31}$.

Before performing free-energy calculations to compute the phase diagram, one must decide which ice phases to consider, bearing in mind that the experimentally confirmed phases may not be exhaustive. Moreover, many ice phases come in pairs of a proton-disordered and proton-ordered form, e.g. $\mathrm{I}_{\mathrm{h}}$ and XI, III and IX, V and XIII, VI and XV, VII and VIII, and XII and XIV ${ }^{32-34}$. Ices III and $\mathrm{V}$ are known to exhibit only partial proton disorder ${ }^{35}$. Both full and partial proton disorder make free-energy calculations more challenging. This is because the enthalpies of different proton-disordered manifestations can be significantly different, but the disorder is not possible to equilibrate at the time scale of molecular dynamics (MD) simulations.

Here, we compute the phase diagram of water at three hybrid DFT levels of theory (revPBE0-D3, PBE0-D3 and B3LYP-D3), accounting for thermal and nuclear fluctuations as well as proton disorder. We start from 50 putative ice crystal structures,

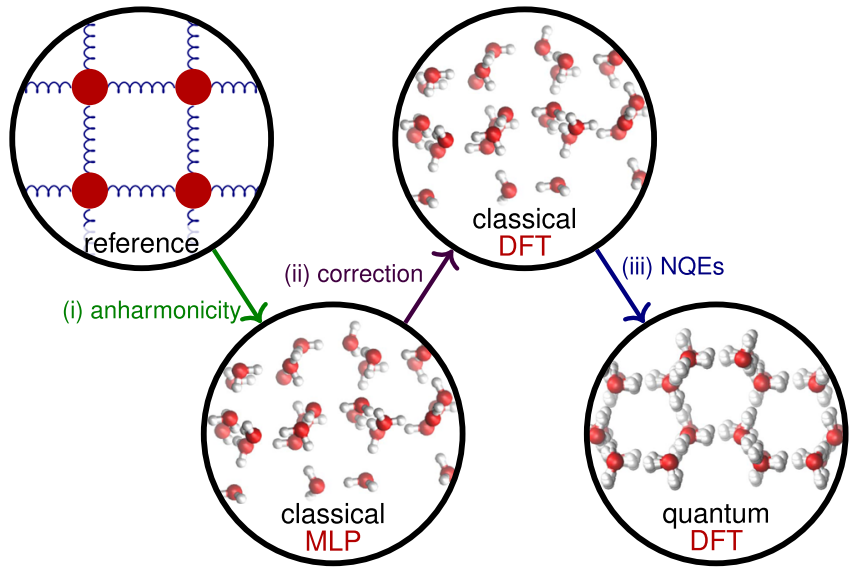

Fig. 1 Schematic illustration of the procedure. Thermodynamic integration steps from a reference system to the classical system described by the MLP, to the classical system described by DFT, and finally to the system with quantum-mechanical nuclei described by DFT.

including all the experimentally known ices. To circumvent the prohibitive cost of ab initio MD simulations, we use a recent $\mathrm{MLP}^{29}$ as a surrogate model when performing free-energy calculations, and then promote the results to the DFT level as well as account for NQEs. This workflow is illustrated in Fig. 1 and described in the 'Methods' section.

\section{Results}

Chemical potentials. For each ice structure, we first obtain the chemical potential over a temperature range of $25 \mathrm{~K}$ to $300 \mathrm{~K}$ and a pressure range of 0 bar to 10,000 bar by performing classical free-energy calculations using the thermodynamic integration (TI) method as described in the 'Methods' section. The MLP employed in these calculations is based on the hybrid revPBE $0^{36}$ functional with a semi-classical D3 dispersion correction ${ }^{37}$. This MLP reproduces many properties of water, including the densities and the relative stabilities of $\mathrm{I}_{\mathrm{h}}, \mathrm{I}_{\mathrm{c}}$ and liquid water at the ambient pressure $^{29}$. Because the MLP is only trained on liquid water and not on either the structures or the energetics of the ice phases, it allows us to explore the ice phase diagram in an agnostic fashion. It nevertheless reproduces lattice energies, molar volumes and phonon densities of states of diverse ice phases, since local atomic environments found in liquid water also cover those observed in the ice phases ${ }^{38}$.

The chemical potentials (expressed per molecule of $\mathrm{H}_{2} \mathrm{O}$ throughout this work) at $225 \mathrm{~K}$ computed using the MLP are shown in Fig. 2a. Focussing only on the phases whose MLP chemical potential is within $10 \mathrm{meV}$ of the ground-state phase under all conditions of interest, we narrow down the selection to 12 ice phases-ices $\mathrm{I}_{\mathrm{h}}, \mathrm{I}_{\mathrm{c}}, \mathrm{II}, \mathrm{III}, \mathrm{V}, \mathrm{VI}, \mathrm{VII}, \mathrm{IX}, \mathrm{XI}, \mathrm{XI}_{\mathrm{c}}$, XIII and $\mathrm{XV}-$ and the liquid. These phases are all known from experiment, which suggests that the experimental phase diagram of water is indeed complete ${ }^{2}$. Moreover, as the chemical potential difference between $I_{h}$ and $I_{c}$ is small and has already been studied in ref. ${ }^{29}$, we report the results only for 'ice I'.

The treatment of proton-disordered phases is complicated because choosing a non-representative configuration can introduce a significant bias in enthalpy and in turn lead to an incorrect phase diagram ${ }^{8}$. To overcome this, we use a combination of the Buch algorithm ${ }^{39}$ and GenIce ${ }^{40}$ to generate 5-8 different depolarised proton-disordered manifestations of each disordered phase considered. For the partially proton-disordered phases III and $\mathrm{V}$, we first construct many configurations, and then only consider the ones that match the experimental site occupancies ${ }^{41}$. 

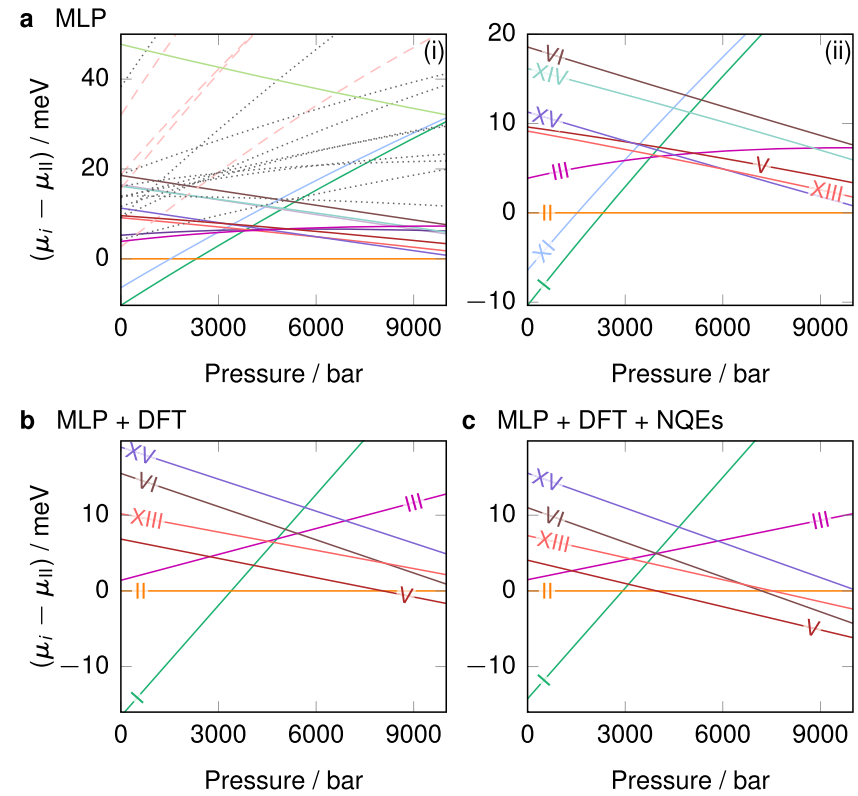

c $\mathrm{MLP}+\mathrm{DFT}+\mathrm{NQES}$

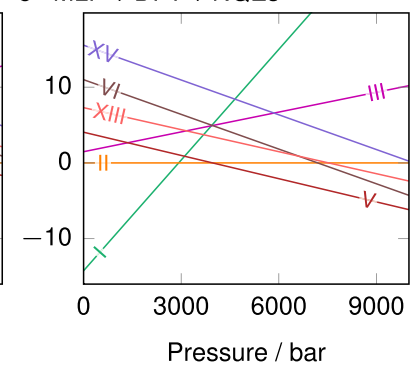

d representative errors in chemical potentials
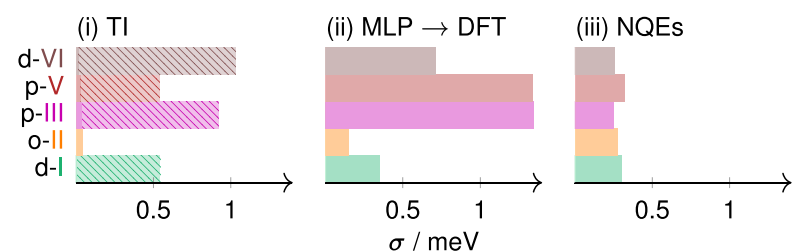

Fig. 2 Computed chemical potentials and error analysis. Per-molecule chemical potentials for the phases (meta)stable at $225 \mathrm{~K}$, shown relative to the chemical potential of ice II. The MLP results are in $\mathbf{a}$. In subpanel (i), solid lines show experimentally known bulk phases, dashed lines show various known low-density structures, and dotted lines show other hypothetical phases. Subpanel (ii) shows the same data, but only for the known experimental phases, as labelled. b Shows the chemical potentials at the level of the revPBEO-D3 DFT for classical systems, and c shows DFT results with NQE corrections. d Illustrates representative standard deviations for selected proton-disordered (' $d$ '), partially proton-disordered $(' p ')$ and proton-ordered ('o') phases. Contributions are split into those arising from (i) the thermodynamic integration to the classical system described by the MLP, with the contribution from proton disorder shown as a lighter hatched area; (ii) the MLP to the revPBEO-D3 correction term; and (iii) the term accounting for NQEs.

We compute the chemical potential of each such configuration independently and average over the results, and finally add a configurational entropy associated with (partial) proton disorder ${ }^{34,35}$. We have assumed that the experimental proton disorder is correct for the potential used, which may not be completely accurate ${ }^{8,42}$, but is the best assumption we can make, since equilibration of proton disorder is not feasible to achieve on computationally tractable time scales.

We perform a free-energy perturbation to promote the MLP results to the hybrid DFT level (see revPBE0-D3 results in Fig. 2b), as detailed in the 'Methods' section. This correction is necessary to recover the true chemical potential at the DFT level, because the MLP inevitably leads to small residual errors ${ }^{43}$. Specifically, proton order leads to long-range electrostatics that can destabilise the solid, but the MLP only accounts for shortrange interactions. Albeit small in absolute terms, the correction to DFT causes a changeover of stability, and in particular reduces the stability of the proton-ordered phases (such as ice II and XV), as can be seen from Fig. 2b. Although the proton-ordered phases
XI, XIII and XV are more stable than their proton-disordered analogues (I, V and VI, respectively) at the MLP level at low temperatures, at the DFT level, the proton order-disorder transition occurs at considerably lower temperatures than we focus on here, and so we do not characterise this transition further, and we do not show the proton-ordered phases XI or XIV in Fig. 2b.

Finally, we consider NQEs by performing path-integral molecular dynamics (PIMD) simulations. In general, NQEs serve to stabilise higher-density phases. For all phases, the degree of stabilisation increases with increasing pressure, and decreases with increasing temperature. Whilst the overall form of the chemical potential plot (Fig. 2c) changes only subtly, NQEs can significantly shift the phase boundaries, and we return to this point below.

Although it is often difficult to determine error bars in freeenergy calculations ${ }^{44}$, we have estimated the errors arising from each step of the calculation (Fig. 2d) following the steps outlined in the 'Methods' section. The uncertainty is small for the protonordered phases, but considerably larger for the proton-disordered phases, in which there are more varied local environments. The typical uncertainty in the chemical potential of each phase is at most $\sim 2.5 \mathrm{meV}$, but, as we show below, even this relatively small difference is often sufficient to change the coexistence lines significantly.

Phase diagram. We determine the phase diagram of water by analysing the computed chemical potentials over a wide range of pressure and temperature conditions. In Fig. 3, we show the phase diagram for the classical system described by the MLP, the ab initio phase diagram including NQEs, as well as the experimental phase diagram for comparison. Even just at the MLP level without NQEs, the phase diagram is already a reasonable approximation (Fig. 3b). It captures the negative gradient of the pressure-temperature coexistence curve between ice I and the liquid, but fails to account for ices III and V, and the proton-ordered ice $\mathrm{XV}$ is more stable than its disordered analogue (VI). As discussed above, the MLP is prone to overestimating the stability of the proton-ordered phases, which leads to this computational artefact.

When the MLP is corrected to the DFT level of theory and NQEs are accounted for, the resulting phase diagram (Fig. 3c) is considerably improved, and is in close agreement with experiment. [The computed chemical potential difference between ice I and water at the same level of theory in ref. ${ }^{29}$ suffered from a flipped sign when adding the $\mu^{\mathrm{MLP} \rightarrow \mathrm{DFT}}$ terms, and the corrected melting point for $\mathrm{H}_{2} \mathrm{O}$ is $274(2) \mathrm{K}$. The error bar on the melting point of the current work is larger at $\sim 6 \mathrm{~K}$, primarily because only 5 different realisations of the proton-disordered ice $I_{h}$ were considered rather than the 16 of ref. ${ }^{29}$.] However, at very high pressure, the coexistence curve between ices V and VI has too steep a gradient compared to experiment. This may arise from the inaccuracy of the reference DFT functionals ${ }^{45}$ or from the finite basis set and energy cutoffs employed in the DFT calculations. To illustrate the effect of employing different DFT approximations, we show analogous phase diagrams for two alternative DFT functionals, B3LYP-D3 and PBE0-D3, in Fig. 3d. The melting points at 1 bar, 274(6) $\mathrm{K}$ and $268(6) \mathrm{K}$, respectively, are somewhat lower than for the revPBE0-D3 functional, although the error bars are comparable with the difference. In terms of the phase diagrams at higher pressures, PBE0-D3 shows better agreement with experiment compared to the B3LYP-D3 functional.

In none of three phase diagrams shown in Fig. $3 c$, $d$ is ice III thermodynamically stable. From the chemical potential results, we can see that ice III is within $\sim 2 \mathrm{meV}$ to the thermodynamically stable phase at pressures around 3000 bar and temperatures 

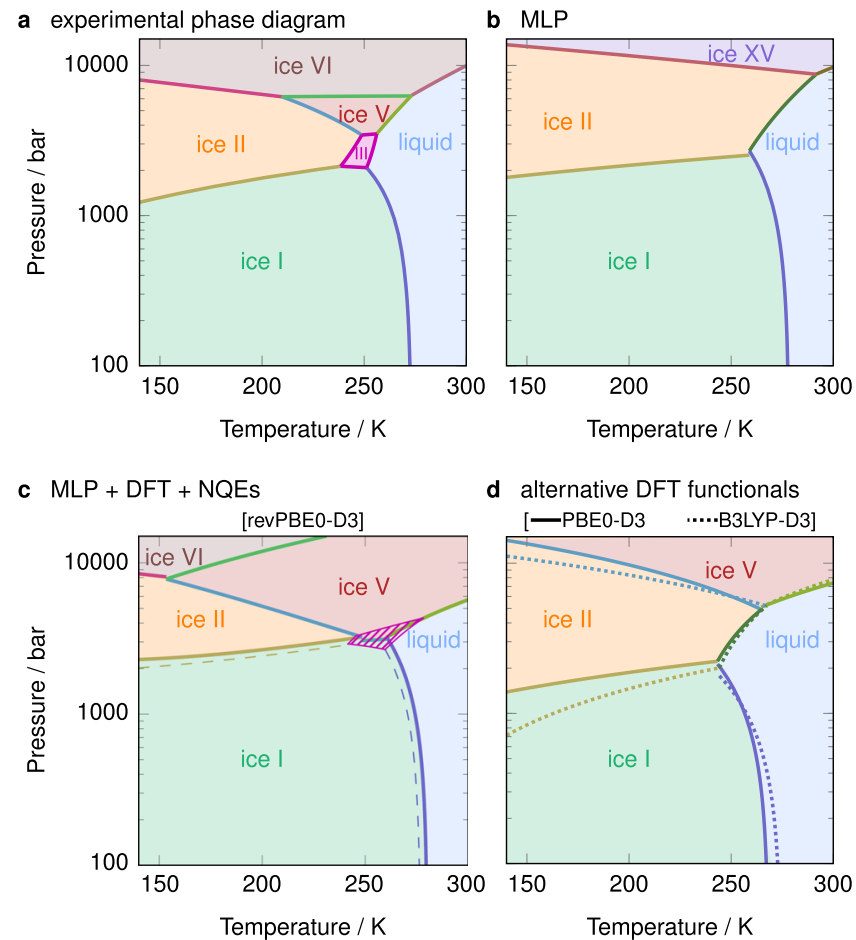

Fig. 3 Comparison of phase diagrams. Phase diagrams a from experiment ${ }^{64,65}$ and $\mathbf{b}$-d from simulation. The phase diagram in b corresponds to the classical system described by the MLP, while $\mathbf{c}$, $\mathbf{d}$ are at the indicated DFT levels and also account for NQE corrections. Dashed lines in c correspond to an alternative calculation where the chemical potential of ice III was decreased and that of ice I was increased by the typical error in chemical potential calculations (Fig. 2d), as discussed in the text. The hatched area corresponds to the region of stability of ice III in this alternative calculation.

around $250 \mathrm{~K}$, where it is known to be stable from experiment. However, its chemical potential depends crucially on the particular manifestation of proton disorder that we choose, and to compute the chemical potentials of the proton-disordered ice phases, we average over a number of independent simulations with different initial proton-disorder configurations. As we have shown in Fig. 2d, the typical error in computations associated with proton-disordered phases is about $2 \mathrm{meV}$. The extent to which such uncertainties affect the computed phase diagram can be significant: we show in Fig. $3 \mathrm{c}$ the phase diagram obtained when ice III is stabilised whilst ice I is destabilised, in each case by changing their chemical potentials by the standard deviation obtained from the above procedure. In such a scenario, ice III is stable over approximately the right range of temperatures and pressures. Such small changes in free energy particularly affect partially proton-disordered phases such as ice III: different partial orderings (i.e. those with different proton site occupancies) may be stable to different extents ${ }^{8,42}$, and the calculated stability thus depends on the specific proton site occupancies considered in addition to the uncertainty in the calculation itself. The main lessons to be drawn from this are that (i) many of the phases are very close in free energy, particularly in the region around $250 \mathrm{~K}$ and $\sim 3000$ bar, which means that even if they are not thermodynamically stable, they are likely to be metastable and hence may be easier to obtain experimentally at these conditions, and (ii) the phase diagram is an extremely sensitive test of the underlying potential: even a very small change in relative stability can drastically affect the phase behaviour.

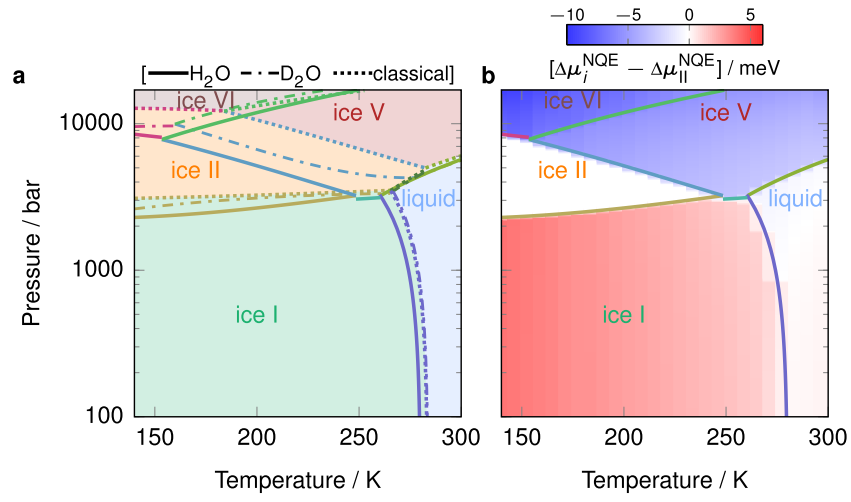

Fig. 4 Phase diagram dependence on NQEs. a Phase diagram excluding NQEs (dotted lines) alongside the full phase diagram (solid lines from Fig. $3 c$ ) and the phase diagram for $D_{2} \mathrm{O}$ (dotted-dashed lines). $\mathbf{b}$ Heat map indicating the correction to the chemical potential of the stable phase $i$ due to NQEs, relative to ice II, overlaid on the phase diagram from Fig. 3c.

Finally, we show the phase diagram with and without accounting for NQEs in Fig. 4a. The NQE correction to the chemical potential of the most stable phase is shown in Fig. 4b; we show the results relative to (metastable) ice II at the same pressure and temperature in order to remove the large effect of the dependence of the mean kinetic energy on the temperature. As we have already discussed, the addition of NQEs to the calculation stabilises the denser phases relative to the less dense ones: ice VI is more stabilised than ice V, ice V more than ice II, ice II more than the liquid, and the liquid more than ice I. We also note that for the solid phases, the density of each phase correlates well with the tetrahedrality parameter studied in this context in ref. ${ }^{22}$.

When NQEs are not accounted for, $\mathrm{H}_{2} \mathrm{O}$ and $\mathrm{D}_{2} \mathrm{O}$ exhibit exactly the same thermodynamic behaviour. However, accounting for the difference between them is necessary for example, for polar ice dating and historical atmospheric temperature estimation $^{46}$, which suggests that a classical description of nuclear motion is not satisfactory. We thus account for NQEs by integrating Eq. (2) to the mass of hydrogen or deuterium to determine the phase behaviour of light and heavy water, respectively. We show the predicted phase diagram of $\mathrm{H}_{2} \mathrm{O}$, $\mathrm{D}_{2} \mathrm{O}$ and 'classical' water (without NQEs) in Fig. 4a. The melting point of ice $\mathrm{I}$ for $\mathrm{H}_{2} \mathrm{O}$ is approximately $4 \mathrm{~K}$ lower than that for $\mathrm{D}_{2} \mathrm{O}$, as investigated previously 29,47 . It is interesting that $\mathrm{D}_{2} \mathrm{O}$ follows almost the same ice I-liquid coexistence line as classical water, since positive and negative contributions to the integral of Eq. (2) largely cancel out ${ }^{29}$. A similar conclusion can be drawn about the coexistence line between ices I and II, perhaps because the density difference between the two phases is similar to that between ice I and the liquid. At higher pressures, however, the phase diagrams of $\mathrm{H}_{2} \mathrm{O}$ and $\mathrm{D}_{2} \mathrm{O}$ are drastically different from classical water, demonstrating the importance of properly accounting for NQEs.

\section{Discussion}

In this work, we have undertaken an exhaustive exploration of the phase diagram of water at three levels of hybrid DFT, including nuclear thermal fluctuations and proton disorder. The agreement between the computed and experimental phase diagrams is very good, but by no means perfect. Part of the difference can be explained in terms of the sensitivity to very small changes in the chemical potentials, which is particularly acute with protondisordered phases. Furthermore, using DFT to model water, even at the hybrid level, has limitations ${ }^{45}$. Finally, it is not guaranteed 
that the phases obtained in experiment are in fact the thermodynamically stable phases; as such, the experimental and the theoretical phase diagram may have subtle differences arising from slightly different definitions of phase stability. Nevertheless, the $a b$ initio phase diagrams show a significant improvement over previous results based on empirical model ${ }^{24,26}$, particularly at low pressure. Moreover, although NQEs are often neglected in the computation of the phase diagram of water, we have properly accounted for them in the present work. This allows us to characterise further the subtle difference between the phase behaviour of $\mathrm{H}_{2} \mathrm{O}$ and $\mathrm{D}_{2} \mathrm{O}$.

Our entire calculation was premised on the fact that a MLP had been parameterised for water ${ }^{29}$, which permitted the study of larger systems for sufficiently long to be able to compute their thermodynamic properties. While the DFT-level phase behaviour we have studied is the best we can do at this stage, for many applications, such as investigating the thermodynamics of the nucleation of ice, even the approach we have followed may not be computationally feasible. It would therefore be very tempting to use the inexpensive MLP on its own in future studies. From our results, we can conclude that, even though the potential was trained solely on liquid-phase structures, the description of the solid phases and the phase behaviour of the MLP on its own is very reasonable. However, a degree of care must be taken in interpreting its results, not least because proton-ordered phases are somewhat too stable when described by the MLP.

The good agreement between the calculated phase diagram and experiment confirms that the hybrid DFT levels of theory describe water well. In fact, the approach we have outlined to compute free energies and in turn phase diagrams provides a particularly difficult benchmark for quantum-mechanical methods. We have shown that three different hybrid DFT functionals (revPBE0-D3, PBE0-D3, B3LYP-D3) result in similar, but certainly not identical, phase behaviour. It would be interesting to apply the same workflow to other electronic structure methods, including random-phase approximation ${ }^{48}$ and DFT at the double hybrid level ${ }^{49}$. Indeed, in the future, one possible way of benchmarking and optimising DFT functionals may well be to evaluate the phase diagram of the material studied.

The present study bridges the gap between electronic structure theory and accurate computation of phase behaviour for water. With a robust framework in place, we aim in future work to investigate the behaviour of ice that is less well understood experimentally. For example, tantalisingly, in Fig. 2a, the chemical potentials of the low-density phases of ice have a large gradient; it would be interesting to determine the phase diagram at negative pressures, which remains rather less well explored ${ }^{50}$. Another intriguing phase transition to study is that between ice VII and liquid water at higher pressures than we have looked at here, which empirical pair potentials do an especially poor job of describing $^{4,51}$. Moreover, the same framework can be used to investigate (meta)stability of novel bulk materials. It further lays the groundwork for utilising experimental phase diagrams to correct and improve interatomic potentials based on electronic structure methods.

\section{Methods}

Candidate ice structures. We start from the 57 phases that were screened from an extensive set of 15,859 hypothetical ice structures using a generalised convex hull construction, an algorithm for identifying promising experimental candidates ${ }^{52,53}$. We eliminate defected phases, dynamically unstable phases, and the very high pressure phase X, but add the originally missing ice IV. In Supplementary Data 1, we provide the structures of the remaining 50 ice phases that we consider in our simulations.
}

DFT calculations. We compute the energies of the water structures using the $\mathrm{CP} 2 \mathrm{~K}$ code ${ }^{54}$ with the revPBE0-D3, the PBE0-D3 and the B3LYP-D3 functionals.
The computational details of the calculations are identical to refs. ${ }^{29,55}$ but for the choice of functionals, and we provide input files in Supplementary Data 1.

Free-energy calculations. We use the method of thermodynamic integration (TI) to compute the Gibbs energy of the selected ice phases at a wide range of thermodynamic conditions, which in turn determine the ice phase diagram. A similar method has previously been used to compute the ice phase diagram using empirical potentials ${ }^{4}$. In our approach, we use a series of steps in simulations of physical or artificial systems to compute the various components of the free-energy difference between a reference system and the fully anharmonic, quantum system, as depicted schematically in Fig. 1.

In the first step of the free-energy calculation for the solid phases, we equilibrate a sample of an ice phase at the temperature and pressure of interest in an isothermal-isobaric simulation with a fluctuating box with a Parrinello-Rahmanlike barostat ${ }^{56}$ to determine the equilibrium lattice parameters at the conditions of interest. We then prepare a perfect crystal with the corresponding lattice parameters and minimise its energy, compute the Helmholtz energy of the reference harmonic crystal by determining the eigenvalues of the hessian matrix, account for the motion of the centre of mass, and finally perform a thermodynamic integration step to the potential of interest in which classical nuclei are described by the MLP [Fig. 1(i)]. By adding a suitable pressure-volume term, we obtain the fully anharmonic classical chemical potential of the ice system described by the MLP. The details of this procedure are discussed in ref. ${ }^{57}$. In this step, we use reasonably large system sizes (of the order a few hundred to several thousand water molecules) to ensure that finite-size effects are minimised. Determining the chemical potential in this way would have been computationally intractable had we employed ab initio calculations, and is only made possible by the use of the MLP. Once the chemical potential is known for the MLP at one set of conditions, we can find it at other pressures and temperatures by numerically integrating the Gibbs-Duhem relation along isotherms and the Gibbs-energy analogue of the Gibbs-Helmholtz relation along isobars, respectively.

To find the free energy of the liquid phase at the MLP level, we perform a series of direct-coexistence simulations ${ }^{44,58}$ of the liquid in contact with ice $\mathrm{I}_{\mathrm{h}}$ at a series of temperatures at 0 bar to determine the coexistence temperature. The melting point $T_{\mathrm{m}}=279.5 \mathrm{~K}$ at 1 bar for the MLP, although evaluated in a different way, is in perfect agreement with a previous simulation that used umbrella sampling ${ }^{29}$. We equate the chemical potentials of the two phases under these conditions and obtain the chemical potential of the liquid at other conditions by thermodynamic integration along isotherms and isobars, as for the ice phases.

In the second step of the procedure [Fig. 1(ii)], we promote the chemical potential of the system as described by the MLP to the DFT potential-energy surface level of theory using a free-energy perturbation,

$$
\Delta \mu^{\mathrm{MLP} \rightarrow \mathrm{DFT}}(P, T)=-\frac{k_{\mathrm{B}} T}{N} \ln \left\langle\exp \left[-\frac{U_{\mathrm{DFT}}-U_{\mathrm{ML}}}{k_{\mathrm{B}} T}\right]\right\rangle_{P, T, \mathcal{H}_{\mathrm{ML}}}
$$

where $\langle\cdot\rangle_{P, T, \mathcal{H}_{\mathrm{ML}}}$ denotes the ensemble average of the system sampled at temperature $T$ and pressure $P$ using the ML hamiltonian $\mathcal{H}_{\mathrm{ML}}$, where $U_{\mathrm{DFT}}$ is the DFT energy and $U_{\mathrm{ML}}$ is the MLP energy, and $N$ is the number of water molecules in the system. This correction is necessary to recover the true chemical potential at the DFT level, in particular to compensate for the lack of long-range electrostatics, which are particularly important in modulating ice phase stabilities ${ }^{59}$. Using the MLP-derived chemical potentials without corrections neglects such long-range contributions and significantly hampers the quantitative accuracy of the computed phase diagram. In practice, we first collect trajectories of ice configurations by performing MD simulations in the isothermal-isobaric ensemble for each promising ice phase using the MLP. The number of water molecules in the simulation cell ranges from 56 to 96 molecules, depending on the unit-cell size of each phase. For each of the proton-disordered (I, VI) or partially disordered phases (III, V), we run independent simulations for 5-8 different depolarised protondisordered configurations. We then select decorrelated configurations from the MD trajectories and recompute their energies at the DFT level. The MLP $\rightarrow$ DFT correction terms are then computed using Eq. (1) and averaged over the different disordered structures for the proton-disordered phases.

In this step, the correction to the chemical potential ranges from $-7.9 \mathrm{meV}$ (for ice VI at $125 \mathrm{~K}$ and $10,000 \mathrm{bar}$ ) to $10.8 \mathrm{meV}$ (for ice $\mathrm{XV}$ at $275 \mathrm{~K}$ and 5000 bar). Ice XV is the proton-ordered analogue of ice VI; similarly, ice XIII, the proton-ordered analogue of ice $\mathrm{V}$, is more stable at the MLP level than it ought to be [e.g. at $225 \mathrm{~K}$ and $7000 \mathrm{bar}$, the corrections to chemical potentials are $\Delta \mu_{\mathrm{V}}=$ $-1.5 \mathrm{meV}$ and $\left.\Delta \mu_{\mathrm{XIII}}=3.4 \mathrm{meV}\right]$. Indeed the difference in the correction term between these two pairs of phases is largely insensitive to temperature and pressure, at least at the pressures where the phases are competitive and for which we have computed the correction reliably; namely, $\Delta \Delta \mu(\mathrm{V} \rightarrow \mathrm{XIII}) \approx 5 \mathrm{meV}$ and $\Delta \Delta \mu(\mathrm{VI} \rightarrow \mathrm{XV}) \approx 11 \mathrm{meV}$.

During the final TI [Fig. 1(iii)], NQEs are taken into account by integrating the quantum centroid virial kinetic energy $\left\langle E_{\mathrm{k}}\right\rangle$ with respect to the fictitious 'atomic' mass $\tilde{m}$ from the classical (i.e. infinite) mass to the physical masses $m^{47,60-63}$. In practice, a change of variable $y=\sqrt{m / \tilde{m}}$ is applied to reduce the discretisation 
error in the evaluation of the integral ${ }^{60}$, yielding

$$
\Delta \mu^{\mathrm{NQE}}(P, T)=2 \int_{0}^{1} \frac{\left\langle E_{\mathrm{k}}\left(1 / y^{2}\right)\right\rangle}{y} \mathrm{~d} y .
$$

We evaluate the integrand using PIMD simulations for $y=1 / 4,1 / 2, \sqrt{2} / 2$ and 1 , and then numerically integrate it. In these PIMD simulations, we use 24 beads for all phases considered at a wide range of constant pressure and temperature conditions for $T \geq 125 \mathrm{~K}$. Relative to ice II, the correction to the chemical potential arising from NQEs ranges from $-8 \mathrm{meV}$ to $5 \mathrm{meV}$.

Uncertainty estimation. To determine the approximate uncertainty associated with the individual chemical potential contributions, we first compute the reference chemical potential of several phases computed from an equilibrated perfect crystal at $125 \mathrm{~K}$ and 0 bar. We repeat this calculation between 5 and 10 times and determine the standard deviation of the resulting chemical potentials. For (partially and fully) proton-disordered phases, we split the contributions to the standard deviation arising from thermodynamic integration, which we obtain by computing the reference chemical potential calculation using the same initial configuration in 5 independent simulations, and from proton disorder, which we obtain by averaging over the chemical potentials arising from several distinct manifestations of the proton disorder in the initial configurations. Similarly, we compute the standard deviation of the correction to the chemical potential when the system is integrated from the MLP to the DFT level evaluated at $225 \mathrm{~K}$ and 3000 bar. For the correction to NQEs, we determine the statistical error in the measurement of the kinetic energy at each scaled mass. We calculate the integral of Eq. (2) with an analogue of Simpson's rule for irregularly spaced data both for the mean values as well as the mean value increased by the standard deviation, and we estimate the a posteriori NQE error to be the difference between these two corrections.

\section{Data availability}

The 50 ice structures studied, along with their chemical potentials, the CP2K input files and a Mathematica notebook for analysis, are provided in Supplementary Data 1 and in the supporting data available at https://doi.org/10.17863/cam.62110 and https://github. com/BingqingCheng/nn-water-phase-diagram.

Received: 4 November 2020; Accepted: 21 December 2020; Published online: 26 January 2021

\section{References}

1. Debenedetti, P. G. Supercooled and glassy water. J. Phys.: Condens. Matter 15, R1669-R1726 (2003)

2. Salzmann, C. G. Advances in the experimental exploration of water's phase diagram. J. Chem. Phys. 150, 060901 (2019).

3. Vega, C., Abascal, J. L. F., Conde, M. M. \& Aragones, J. L. What ice can teach us about water interactions: a critical comparison of the performance of different water models. Faraday Discuss. 141, 251-276 (2009).

4. Vega, C. \& Abascal, J. L. F. Simulating water with rigid non-polarizable models: a general perspective. Phys. Chem. Chem. Phys. 13, 19663-19688 (2011).

5. Noya, E. G., Menduiña, C., Aragones, J. L. \& Vega, C. Equation of state, thermal expansion coefficient, and isothermal compressibility for ices $\mathrm{I}_{\mathrm{h}}, \mathrm{II}$, III, V, and VI, as obtained from computer simulation. J. Phys. Chem. C. 111, 15877-15888 (2007).

6. Abascal, J. L. F., Sanz, E. \& Vega, C. Triple points and coexistence properties of the dense phases of water calculated using computer simulation. Phys. Chem. Chem. Phys. 11, 556-562 (2009).

7. Agarwal, M., Alam, M. P. \& Chakravarty, C. Thermodynamic, diffusional, and structural anomalies in rigid-body water models. J. Phys. Chem. B 115, 6935-6945 (2011).

8. Conde, M. M., Gonzalez, M. A., Abascal, J. L. F. \& Vega, C. Determining the phase diagram of water from direct coexistence simulations: The phase diagram of the TIP4P/2005 model revisited. J. Chem. Phys. 139, 154505 (2013).

9. Quigley, D. \& Rodger, P. M. Metadynamics simulations of ice nucleation and growth. J. Chem. Phys. 128, 154518 (2008).

10. Reinhardt, A., Doye, J. P. K., Noya, E. G. \& Vega, C. Local order parameters for use in driving homogeneous ice nucleation with all-atom models of water. J. Chem. Phys. 137, 194504 (2012).

11. Reinhardt, A. \& Doye, J. P. K. Note: Homogeneous TIP4P/2005 ice nucleation at low supercooling. J. Chem. Phys. 139, 096102 (2013).

12. Malkin, T. L., Murray, B. J., Brukhno, A. V., Anwar, J. \& Salzmann, C. G. Structure of ice crystallized from supercooled water. Proc. Natl Acad. Sci. USA 109, 1041-1045 (2012).
13. Espinosa, J. R., Sanz, E., Valeriani, C. \& Vega, C. Homogeneous ice nucleation evaluated for several water models. J. Chem. Phys. 141, 18C529 (2014)

14. Jorgensen, W. L., Chandrasekhar, J., Madura, J. D., Impey, R. W. \& Klein, M. L. Comparison of simple potential functions for simulating liquid water. $J$. Chem. Phys. 79, 926-935 (1983).

15. Rick, S. W. A reoptimization of the five-site water potential (TIP5P) for use with Ewald sums. J. Chem. Phys. 120, 6085-6093 (2004).

16. Abascal, J. L. F. \& Vega, C. A general purpose model for the condensed phases of water: TIP4P/2005. J. Chem. Phys. 123, 234505 (2005).

17. Berendsen, H. J. C., Grigera, J. R. \& Straatsma, T. P. The missing term in effective pair potentials. J. Phys. Chem. 91, 6269-6271 (1987).

18. Habershon, S. \& Manolopoulos, D. E. Thermodynamic integration from classical to quantum mechanics. J. Chem. Phys. 135, 224111 (2011).

19. Habershon, S. \& Manolopoulos, D. E. Free energy calculations for a flexible water model. Phys. Chem. Chem. Phys. 13, 19714-19727 (2011).

20. Habershon, S., Markland, T. E. \& Manolopoulos, D. E. Competing quantum effects in the dynamics of a flexible water model. J. Chem. Phys. 131, 024501 (2009).

21. McBride, C., Vega, C., Noya, E. G., Ramírez, R. \& Sesé, L. M. Quantum contributions in the ice phases: the path to a new empirical model for waterTIP4PQ/2005. J. Chem. Phys. 131, 024506 (2009).

22. McBride, C., Noya, E. G., Aragones, J. L., Conde, M. M. \& Vega, C. The phase diagram of water from quantum simulations. Phys. Chem. Chem. Phys. 14, 10140-10146 (2012)

23. Reddy, S. K. et al. On the accuracy of the MB-pol many-body potential for water: Interaction energies, vibrational frequencies, and classical thermodynamic and dynamical properties from clusters to liquid water and ice. J. Chem. Phys. 145, 194504 (2016).

24. Wang, L.-P. et al. Systematic improvement of a classical molecular model of water. J. Phys. Chem. B 117, 9956-9972 (2013).

25. Vega, C., Sanz, E. \& Abascal, J. L. F. The melting temperature of the most common models of water. J. Chem. Phys. 122, 114507 (2005)

26. Vega, C., Abascal, J. L. F., Sanz, E., MacDowell, L. G. \& McBride, C. Can simple models describe the phase diagram of water? J. Phys.: Condens. Matter 17, S3283 (2005).

27. Deringer, V. L., Caro, M. A. \& Csányi, G. Machine learning interatomic potentials as emerging tools for materials science. Adv. Mater. 31, 1902765 (2019).

28. Morawietz, T., Singraber, A., Dellago, C. \& Behler, J. How van der Waals interactions determine the unique properties of water. Proc. Natl Acad. Sci. USA 113, 8368-8373 (2016).

29. Cheng, B., Engel, E. A., Behler, J., Dellago, C. \& Ceriotti, M. Ab initio thermodynamics of liquid and solid water. Proc. Natl Acad. Sci. USA 116, 1110-1115 (2019)

30. Niu, H., Bonati, L., Piaggi, P. M. \& Parrinello, M. Ab initio phase diagram and nucleation of gallium. Nat. Commun. 11, 2654 (2020).

31. Cheng, B., Mazzola, G., Pickard, C. J. \& Ceriotti, M. Evidence for supercritical behaviour of high-pressure liquid hydrogen. Nature 585, 217-220 (2020).

32. Pauling, L. The structure and entropy of ice and of other crystals with some randomness of atomic arrangement. J. Am. Chem. Soc. 57, 2680-2684 (1935).

33. Bernal, J. D. \& Fowler, R. H. A theory of water and ionic solution, with particular reference to hydrogen and hydroxyl ions. J. Chem. Phys. 1, 515-548 (1933).

34. Herrero, C. P. \& Ramírez, R. Configurational entropy of hydrogen-disordered ice polymorphs. J. Chem. Phys. 140, 234502 (2014).

35. MacDowell, L. G., Sanz, E., Vega, C. \& Abascal, J. L. F. Combinatorial entropy and phase diagram of partially ordered ice phases. J. Chem. Phys. 121, 10145-10158 (2004)

36. Goerigk, L. \& Grimme, S. A thorough benchmark of density functional methods for general main group thermochemistry, kinetics, and noncovalent interactions. Phys. Chem. Chem. Phys. 13, 6670-6688 (2011).

37. Grimme, S., Hansen, A., Brandenburg, J. G. \& Bannwarth, C. Dispersioncorrected mean-field electronic structure methods. Chem. Rev. 116, 5105-5154 (2016)

38. Monserrat, B., Brandenburg, J. G., Engel, E. A. \& Cheng, B. Liquid water contains the building blocks of diverse ice phases. Nat. Commun.11, 5757 (2020).

39. Buch, V., Sandler, P. \& Sadlej, J. Simulations of $\mathrm{H}_{2} \mathrm{O}$ solid, liquid, and clusters, with an emphasis on ferroelectric ordering transition in hexagonal ice. J. Phys. Chem. B 102, 8641-8653 (1998).

40. Matsumoto, M., Yagasaki, T. \& Tanaka, H. GenIce: Hydrogen-disordered ice generator. J. Comput. Chem. 39, 61-64 (2017).

41. Lobban, C., Finney, J. L. \& Kuhs, W. F. The structure and ordering of ices III and V. J. Chem. Phys. 112, 7169-7180 (2000).

42. Aragones, J. L., MacDowell, L. G. \& Vega, C. Dielectric constant of ices and water: a lesson about water interactions. J. Phys. Chem. A 115, 5745-5758 (2011). 
43. Behler, J. Constructing high-dimensional neural network potentials: a tutorial review. Int. J. Quantum Chem. 115, 1032-1050 (2015).

44. Vega, C., Sanz, E., Abascal, J. L. F. \& Noya, E. G. Determination of phase diagrams via computer simulation: methodology and applications to water, electrolytes and proteins. J. Phys.: Condens. Matter 20, 153101 (2008).

45. Gillan, M. J., Alfè, D. \& Michaelides, A. Perspective: how good is DFT for water? J. Chem. Phys. 144, 130901 (2016).

46. Dreschhoff, G., Jungner, H. \& Laird, C. M. Deuterium-hydrogen ratios, electrical conductivity and nitrate for high-resolution dating of polar ice cores. Tellus B Chem. Phys. Meteorol. 72, 1-6 (2020).

47. Ramírez, R. \& Herrero, C. P. Quantum path integral simulation of isotope effects in the melting temperature of ice Ih. J. Chem. Phys. 133, 144511 (2010).

48. Langreth, D. C. \& Perdew, J. P. Exchange-correlation energy of a metallic surface: Wave-vector analysis. Phys. Rev. B 15, 2884-2901 (1977).

49. Martin, J. M. L. \& Santra, G. Empirical double-hybrid density functional theory: a 'third way' in between WFT and DFT. Isr. J. Chem. 60, 787-804 (2019).

50. Huang, Y. et al. A new phase diagram of water under negative pressure: the rise of the lowest-density clathrate s-III. Sci. Adv. 2, e1501010 (2016).

51. Aragones, J., Conde, M., Noya, E. \& Vega, C. The phase diagram of water at high pressures as obtained by computer simulations of the TIP4P/2005 model: The appearance of a plastic crystal phase. Phys. Chem. Chem. Phys. 11, 543-555 (2009).

52. Anelli, A., Engel, E. A., Pickard, C. J. \& Ceriotti, M. Generalized convex hull construction for materials discovery. Phys. Rev. Mater. 2, 103804 (2018).

53. Engel, E. A., Anelli, A., Ceriotti, M., Pickard, C. J. \& Needs, R. J. Mapping uncharted territory in ice from zeolite networks to ice structures. Nat. Commun. 9, 2173 (2018).

54. Lippert, G., Hutter, J. \& Parrinello, M. The Gaussian and augmented-planewave density functional method for ab initio molecular dynamics simulations. Theor. Chem. Acc. 103, 124 (1999).

55. Marsalek, O. \& Markland, T. E. Quantum dynamics and spectroscopy of ab initio liquid water: the interplay of nuclear and electronic quantum effects. J. Phys. Chem. Lett. 8, 1545 (2017).

56. Parrinello, M. \& Rahman, A. Polymorphic transitions in single crystals: a new molecular dynamics method. J. Appl. Phys. 52, 7182-7190 (1981).

57. Cheng, B. \& Ceriotti, M. Computing the absolute Gibbs free energy in atomistic simulations: applications to defects in solids. Phys. Rev. B 97, 054102 (2018).

58. Opitz, A. C. L. Molecular dynamics investigation of a free surface of liquid argon. Phys. Lett. A 47, 439-440 (1974).

59. Melko, R. G., den Hertog, B. C. \& Gingras, M. J. Long-range order at low temperatures in dipolar spin ice. Phys. Rev. Lett. 87, 067203 (2001).

60. Ceriotti, M. \& Markland, T. E. Efficient methods and practical guidelines for simulating isotope effects. J. Chem. Phys. 138, 014112 (2013).

61. Cheng, B., Behler, J. \& Ceriotti, M. Nuclear quantum effects in water at the triple point: using theory as a link between experiments. J. Phys. Chem. Lett. 7, 2210-2215 (2016).

62. Cheng, B. \& Ceriotti, M. Direct path integral estimators for isotope fractionation ratios. J. Chem. Phys. 141, 244112 (2014).

63. Cheng, B., Paxton, A. T. \& Ceriotti, M. Hydrogen diffusion and trapping in airon: the role of quantum and anharmonic fluctuations. Phys. Rev. Lett. 120, 225901 (2018).
64. León, G., Romo, S. R. \& Tchijov, V. Thermodynamics of high-pressure ice polymorphs: ice II. J. Phys. Chem. Solids 63, 843-851 (2002).

65. Wagner, W., Riethmann, T., Feistel, R. \& Harvey, A. H. New equations for the sublimation pressure and melting pressure of $\mathrm{H}_{2} \mathrm{O}$ ice $\mathrm{I}_{\mathrm{h}}$. J. Phys. Chem. Ref. Data 40, 43103 (2011).

\section{Acknowledgements}

We thank Jan Gerit Brandenburg and Carlos Vega for reading an early draft and providing constructive and useful comments and suggestions. A.R. and B.C. acknowledge resources provided by the Cambridge Tier-2 system operated by the University of Cambridge Research Computing Service funded by EPSRC Tier-2 capital grant EP/P020259/1. B.C. acknowledges funding from the Swiss National Science Foundation (Project P2ELP2-184408), and allocation of CPU hours by CSCS under Project ID s957.

\section{Author contributions}

A.R. and B.C. designed and performed the research, analysed data and wrote the paper.

\section{Competing interests}

The authors declare no competing interests.

\section{Additional information}

Supplementary information is available for this paper at https://doi.org/10.1038/s41467 020-20821-w.

Correspondence and requests for materials should be addressed to A.R. or B.C.

Peer review information Nature Communications thanks the anonymous reviewer(s) for their contribution to the peer review of this work. Peer reviewer reports are available.

Reprints and permission information is available at http://www.nature.com/reprints

Publisher's note Springer Nature remains neutral with regard to jurisdictional claims in published maps and institutional affiliations.

Open Access This article is licensed under a Creative Commons Attribution 4.0 International License, which permits use, sharing, adaptation, distribution and reproduction in any medium or format, as long as you give appropriate credit to the original author(s) and the source, provide a link to the Creative Commons license, and indicate if changes were made. The images or other third party material in this article are included in the article's Creative Commons license, unless indicated otherwise in a credit line to the material. If material is not included in the article's Creative Commons license and your intended use is not permitted by statutory regulation or exceeds the permitted use, you will need to obtain permission directly from the copyright holder. To view a copy of this license, visit http://creativecommons.org/ licenses/by/4.0/.

(C) The Author(s) 2021 